2012

\title{
Reconnection Events In Two-Dimensional Hall Magnetohydrodynamic Turbulence
}

S. Donato

S. Servidio

P. Dmitruk

V. Carbone

M. A. Shay

See next page for additional authors

Follow this and additional works at: https://researchrepository.wvu.edu/faculty_publications

\section{Digital Commons Citation}

Donato, S.; Servidio, S.; Dmitruk, P.; Carbone, V.; Shay, M. A.; Cassak, P. A.; and Matthaeus, W. H., "Reconnection Events In TwoDimensional Hall Magnetohydrodynamic Turbulence" (2012). Faculty Scholarship. 657.

https://researchrepository.wvu.edu/faculty_publications/657 
Authors

S. Donato, S. Servidio, P. Dmitruk, V. Carbone, M. A. Shay, P. A. Cassak, and W. H. Matthaeus 


\title{
Reconnection events in two-dimensional Hall magnetohydrodynamic turbulence
}

\author{
S. Donato, ${ }^{1}$ S. Servidio, ${ }^{1}$ P. Dmitruk, ${ }^{2}$ V. Carbone,${ }^{1}$ M. A. Shay, ${ }^{3}$ P. A. Cassak, ${ }^{4}$ \\ and W. H. Matthaeus ${ }^{3}$ \\ ${ }^{1}$ Dipartimento di Fisica, Università della Calabria, I-87036 Cosenza, Italy \\ ${ }^{2}$ Departamento de Física, Facultad de Ciencias Exactas y Naturales, Universidad de Buenos Aires \\ and Instituto de Física de Buenos Aires, CONICET, Buenos Aires, Argentina \\ ${ }^{3}$ Bartol Research Institute and Department of Physics and Astronomy, University of Delaware, Newark, \\ Delaware 19716, USA \\ ${ }^{4}$ Department of Physics, West Virginia University, Morgantown, West Virginia 26506, USA
}

(Received 28 February 2012; accepted 6 September 2012; published online 21 September 2012)

\begin{abstract}
The statistical study of magnetic reconnection events in two-dimensional turbulence has been performed by comparing numerical simulations of magnetohydrodynamics (MHD) and Hall magnetohydrodynamics (HMHD). The analysis reveals that the Hall term plays an important role in turbulence, in which magnetic islands simultaneously reconnect in a complex way. In particular, an increase of the Hall parameter, the ratio of ion skin depth to system size, broadens the distribution of reconnection rates relative to the MHD case. Moreover, in HMHD the local geometry of the reconnection region changes, manifesting bifurcated current sheets and quadrupolar magnetic field structures in analogy to laminar studies, leading locally to faster reconnection processes in this case of reconnection embedded in turbulence. This study supports the idea that the global rate of energy dissipation is controlled by the large scale turbulence, but suggests that the distribution of the reconnection rates within the turbulent system is sensitive to the microphysics at the reconnection sites. @ 2012 American Institute of Physics. [http://dx.doi.org/10.1063/1.4754151]
\end{abstract}

\section{INTRODUCTION}

Magnetic reconnection is a fundamental phenomenon in magnetized plasmas, responsible for magnetic energy release, topology change, and particle energization, and therefore it is of widespread relevance in astrophysical and laboratory systems. ${ }^{1-3}$ The problem of magnetic reconnection has been investigated mostly assuming simplified geometries and well known boundary (and initial) conditions, but, since it might occur in any region separating distinct magnetic topologies, it is expected to be of importance in turbulence. Recently, ${ }^{4,5}$ it has been proposed that magnetohydrodynamic (MHD) turbulence provides a kind of unbiased and natural local boundary condition for reconnection, producing much faster reconnection events than one would expect in laminar regimes.

Besides MHD turbulence, ${ }^{7}$ another ingredient that may accelerate the process of reconnection is the Hall effect. ${ }^{8}$ The latter is always important for dynamical processes that occur at scales comparable to the ion skin depth (or ion inertial length), defined as $d_{i}=c / \omega_{p i}$ ( $\omega_{p i}$ being the ion plasma frequency). The Hall effect becomes globally important and even dominant as the ion skin depth becomes comparable to the system size $L_{0}$, namely, when $d_{i} / L_{0} \neq 0 .{ }^{9}$ Generally, the Hall effect is thought to be fundamental for astrophysical plasmas, since it modifies small scale turbulent activity, producing a departure from MHD predictions. ${ }^{10-14}$ In the past years, the role of the ion skin depth on reconnection has been matter of numerous numerical investigations. ${ }^{15-18}$ In particular, it has been proposed that the Hall term in resistive plasmas causes a catastrophic release of magnetic energy, leading to fast magnetic reconnection onset, ${ }^{19,20}$ with reconnection rates faster than the Sweet-Parker expectation.

In this manuscript, we combine the above ideas, namely that reconnection is locally enhanced by both MHD turbulence and by the Hall effect, investigating the statistics of magnetic reconnection in 2D Hall magnetohydrodynamic (HMHD) turbulence. Using high resolution pseudo-spectral numerical simulations, we will compare the statistical properties of reconnection in MHD and HMHD turbulence, by comparing a sequence of simulations with increasing strength of the Hall effect. We find a broader range of reconnection rates (normalized to the root-mean-square magnetic field) with respect the MHD case, and faster reconnection processes. The introduction of the Hall effect affects as well the local geometry of the reconnection regions, producing bifurcations in the current sheets and a quadrupolar structure of the magnetic field.

The outline of the paper is as follows: In Sec. II, the incompressible HMHD equations are introduced together with the numerical method employed to solve the equations. A global overview of the MHD turbulence properties for all the simulations performed is given as well. The comparison between the MHD and the HMHD simulations, together with the new features produced by the Hall physics, is presented in Sec. III. In Sec. IV, the conclusions are given, and possible implications for turbulent astrophysical plasmas are discussed. The importance of numerical accuracy will be discussed in the Appendix.

\section{NUMERICAL SIMULATIONS OF TURBULENCE}

The equations of incompressible HMHD can be written in Alfvén units, with lengths scaled to $L_{0}$, and times to a 
characteristic Alfvén time $\tau_{A}$. In 2.5D (three components of the vector fields with spatial variations in two directions) the equations read

$$
\begin{gathered}
\frac{\partial \boldsymbol{v}}{\partial t}=-(\boldsymbol{v} \cdot \nabla) \boldsymbol{v}-\nabla P+\boldsymbol{j} \times \boldsymbol{b}+R_{\nu}^{-1} \nabla^{2} \boldsymbol{v}, \\
\frac{\partial \boldsymbol{b}}{\partial t}=\nabla \times\left[\left(\mathbf{v}-\epsilon_{H} \boldsymbol{j}\right) \times \boldsymbol{b}\right]+R_{\mu}^{-1} \nabla^{2} \boldsymbol{b},
\end{gathered}
$$

where $\boldsymbol{v}$ is the velocity field and $\boldsymbol{b}$ is the magnetic field. Both fields can be decomposed into perpendicular (in-plane) and parallel (out-of-plane, along $z$ ) components, namely $\boldsymbol{b}=\left(\boldsymbol{b}_{\perp}, b_{z}\right)$ and $\boldsymbol{v}=\left(\boldsymbol{v}_{\perp}, v_{z}\right)$. For the in-plane magnetic field, $\boldsymbol{b}_{\perp}=\nabla a \times \hat{z}$. Here $a$ is the magnetic potential (or magnetic flux function) which contains important information about the topology of the magnetic field and the nature of reconnection. No external mean field has been imposed. In Eqs. (1) and (2), $\boldsymbol{j}=\nabla \times \boldsymbol{b}$ is the current density (note that $j_{z}=-\nabla^{2} a$ ) and $P$ is the pressure that is determined by the solenoidal condition $\nabla \cdot v=0$. The parameters $R_{\mu}$ and $R_{\nu}$ are the magnetic and the kinetic Reynolds numbers, respectively. The coefficient $\epsilon_{H}=d_{i} / L_{0}$ is the Hall parameter, providing an explicit measure of the importance of the Hall term in Ohm's law. Note that, for $\epsilon_{H} \rightarrow 0$, Eqs. (1) and (2) reduce to MHD. Generally speaking, the Hall term becomes a significant factor at wavenumbers $k$ such that $k L_{0} \epsilon_{H}=k d_{i} \sim 1$.

Equations (1) and (2) are solved in double periodic $(x, y)$ Cartesian geometry, with a box size of $2 \pi L_{0}$, using $4096^{2}$ grid points, and with $R_{\mu}=R_{\nu}=1700$. We use a well-tested and accurate pseudo-spectral code, fully dealiased with a 2/3-rule. We fix the above parameters for all the simulations reported here and we vary $\epsilon_{H}$, going from the MHD case $\left(\epsilon_{H}=0\right)$ to the Hall case, choosing $\epsilon_{H}=1 / 400,1 / 100,1 / 50$. For all the runs, the energy is initially concentrated in the shells with $4 \leq k \leq 10$ (wavenumbers $k$ in units of $1 / L_{0}$ ) with mean value $E=(1 / 2)\left\langle|\mathbf{v}|^{2}+|\mathbf{b}|^{2}\right\rangle \simeq 1$, where $\langle\ldots\rangle$ indicates a volume average. Using the above set of parameters, the dissipation wavenumber is $k_{\text {diss }}=R_{\mu}^{1 / 2}\left\langle j^{2}\right\rangle^{1 / 4} \sim 200$. For the HMHD simulations, the Hall wavenumbers are $k_{H}=\epsilon_{H}^{-1}=400,100,50$. The maximum resolved wavenumber in all the simulations (allowed by the simulation resolution and the $2 / 3$ rule) is $k_{\max }=4096 / 3 \approx 1365$. A summary of the simulations is reported in Table I. Hereafter, we will call each simulation with its relative roman number, as they are labeled in Table I.

We perform our analysis at a fixed time of the turbulent evolution, considering the state of the system when the mean

TABLE I. Table of parameters of the runs. The second column is the resolution of the simulation, third column the Reynolds numbers, fourth column reports the dissipative scale of the system, and the last column shows the Hall parameter.

\begin{tabular}{lccccc}
\hline \hline Run & Eqs. & Resolution & $R_{\mu}\left(R_{\nu}\right)$ & $\lambda_{\text {diss }}=1 / k_{\text {diss }}$ & $\epsilon_{H}\left(k_{H}^{-1}\right)$ \\
\hline I & MHD & $4096^{2}$ & 1700 & $1 / 196$ & 0 \\
II & HMHD & $4096^{2}$ & 1700 & $1 / 195$ & $1 / 400$ \\
III & HMHD & $4096^{2}$ & 1700 & $1 / 188$ & $1 / 100$ \\
IV & HMHD & $4096^{2}$ & 1700 & $1 / 179$ & $1 / 50$ \\
\hline \hline
\end{tabular}

square current density $\left\langle j_{z}^{2}\right\rangle$ is very near to its peak value. At this time, in fact, the peak of small scale turbulent activity is achieved. This characteristic time is the same for all the runs performed here, namely $t \sim 0.5 \tau_{A}$. One way to quantify the differences between MHD and HMHD turbulence is to compute the power spectra for $\boldsymbol{b}_{\perp}$ and $\boldsymbol{v}_{\perp}$ (in-plane components), the former is plotted in Fig. 1. We remark that the case with $k_{H}=400$ (run II) shows no appreciable difference from MHD (run I). This is due to the fact that the Hall effect becomes significant in this case in the dissipation range, and not in the inertial range, since in this simulation, $k_{\text {diss }}<k_{H}$ (see Table I). In contrast, runs III and IV clearly differ from the MHD case for wavenumbers $>k_{H}$. This difference in the power spectra has been already noticed in previous works, and is generally attributed to the dispersive effects. These effects can break, in fact, the Alfvénic correlations that are typical of MHD. ${ }^{10,21}$

When the turbulence is fully developed, coherent structures appear. They can be identified as magnetic islands (or flux tubes in 3D) that differ in size and energy. Between these interacting islands the perpendicular (out-of-plane) component of the current density $j_{z}$ becomes very large, as can be seen from Fig. 2, where a comparison between MHD and HMHD is shown. This spatial "burstiness" of the current is related to the intermittent nature of the magnetic field. In fact, like the velocity field in hydrodynamics, both the magnetic and velocity fields in MHD show a strong tendency to generate increasing levels of phase coherence at smaller scales. ${ }^{22}$ From a comparison between run I and run IV, we observe differences concerning the shape of current sheets: in the Hall case the current filaments display a bifurcated structure. We will come to this point in a later discussion. Another interesting feature is that the HMHD current sheets are shorter and thinner. This is reminiscent of the systematic shortening and thinning of current sheets seen in isolated laminar reconnection simulations. ${ }^{23}$ In order to further investigate this interesting phenomenon, in Sec. III, we will carry out local analysis of the reconnection events.

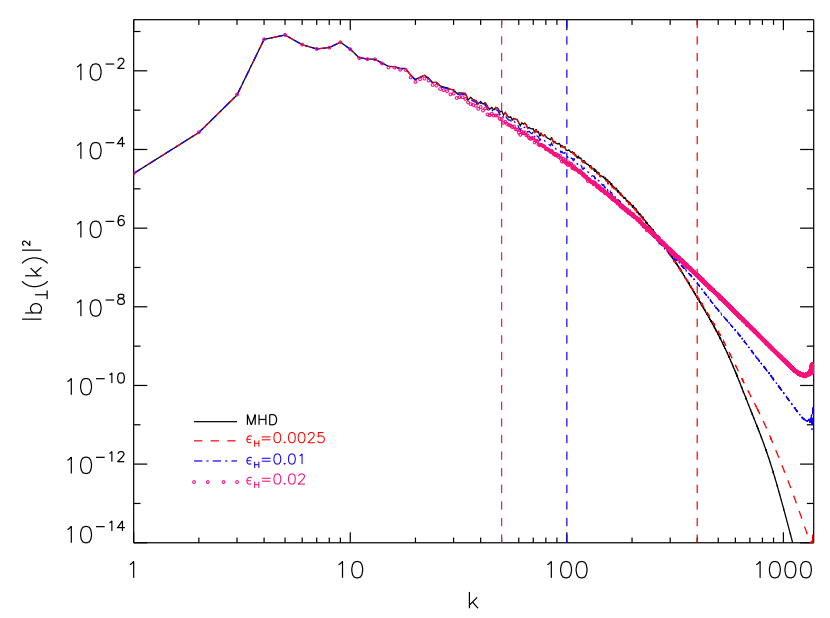

FIG. 1. Power spectra of the "perpendicular" magnetic energy, for all the runs reported in Table I. The vertical dashed lines represent the Hall $k$-vector for runs II, III, and IV, that is, respectively, $k_{H}=400$ (red), $k_{H}=100$ (blue), and $k_{H}=50$ (magenta). 

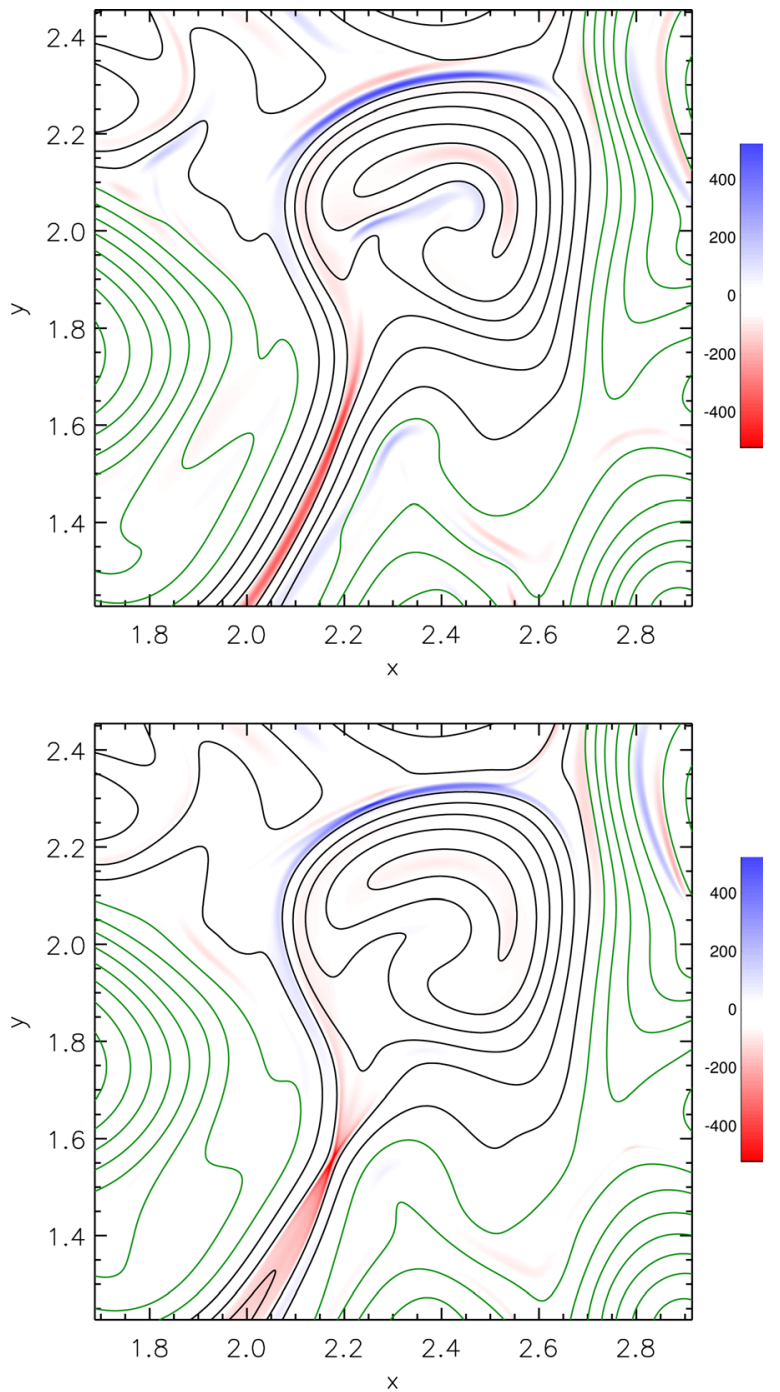

FIG. 2. Current density profile $j_{z}$ (shaded contour) in a small sub-region of the simulation box, for both MHD (top) and HMHD with $\epsilon_{H}=1 / 50$ (bottom). The magnetic flux $a$ is also represented (line contour). As expected in 2D turbulence, strong and narrow peaks of current densities are present between magnetic islands. As can be seen in Fig. 3, current density is higher in HMHD.

The current density $j_{z}$ is an important quantity since it develops small scale features in both turbulence and in reconnection. We show in Fig. 3 the probability distribution function (PDF) of $j_{z}$, for each run in Table I. The core of the distributions is very similar for all the simulations, but, in the HMHD cases, the tails are more pronounced. This implies that the Hall effect causes an enhancement of the small scale activity that is responsible for increasing intermittency in the system. We now examine the quantitative connection between enhanced intermittency and reconnection rates.

\section{RECONNECTION IN TURBULENCE: HMHD vs. MHD}

As reported in Fig. 4, the magnetic potential $a$ reveals a collection of magnetic islands with different size and shape. See Figs. 2 and 4, for example, from two runs. Very similar patterns are observed for all the runs. Note that the potential $a$ is very similar in both cases, since this field is generally large-scale and very smooth. To capture the influence of the

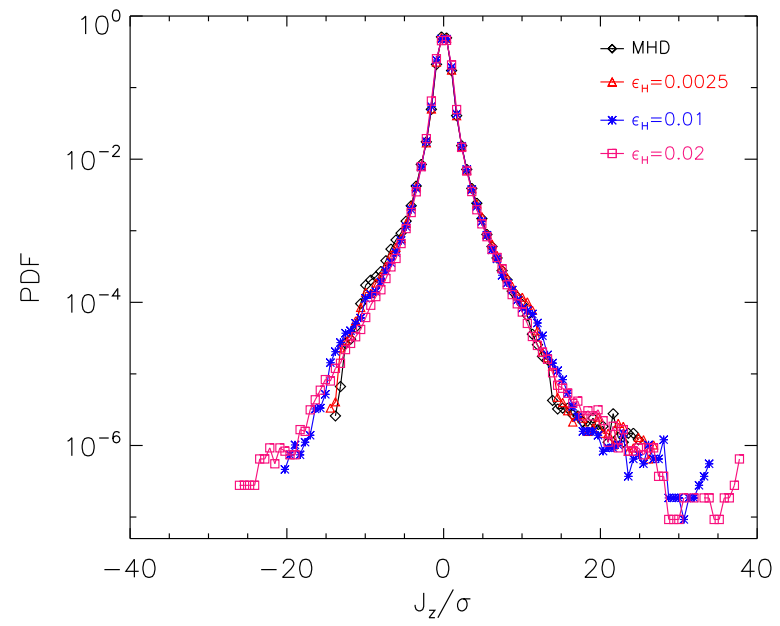

FIG. 3. PDF of $j_{z}$, normalized to its own $r m s$ value, for $\epsilon_{H}=0$ (black), $1 / 400$ (red), 1/100 (blue), and 1/50 (magenta). The longer tails present in run IV may be the signature of more intense small-scale activity, due to stronger dispersive effects.

Hall physics, one should look at the local structure of the current, shown in Fig. 5 (top). When the Hall effect is significant, a clear bifurcation of current sheets is observed.

To further investigate the role of the Hall effect on the process of reconnection in turbulence, we analyze the out-ofplane magnetic field $b_{z}$ around some $X$-points. As expected from theory, ${ }^{8}$ an out-of-plane magnetic quadrupole forms nearby reconnection sites; this is shown in Fig. 5 (bottom). The magnetic field shows four distinctive polarities, organized with respect to the X-point. This effect is thought to be a strong signature of Hall activity during reconnection in astrophysical plasmas, ${ }^{24}$ and in laboratory plasmas. ${ }^{25,26}$ Here we confirm that this is a clear signature of Hall effect in turbulent reconnection.

In order to understand quantitatively the Hall effect on reconnection, we analyze the magnetic field topology and reconnection rates. We begin by inspection of $a(\boldsymbol{x})$, studying its square Hessian matrix, defined as $H_{i, j}(\boldsymbol{x})=\frac{\partial^{2} a}{\partial x_{i} \partial x_{j}}$. At each neutral point, where $\nabla a=0$, we compute the eigenvalues of $H_{i, j}$. If both eigenvalues are positive (negative), the point is a local minimum (maximum) of $a$ (an $\mathrm{O}$ point). If the eigenvalues are of mixed sign, it is a saddle point (an $\mathrm{X}$ point). See Refs. 4-6 for more details on this standard analysis. In Fig. 4, an example of a magnetic potential landscape together with its critical points is reported for a subregion within the MHD and an HMHD simulation. The number of X-points is $\sim 127$, and is a similar number for all the runs. Once we have obtained the position of all the critical points, it is possible to measure the reconnection rate of interacting islands as the rate of change of the magnetic flux at each X-point:

$$
\left.\frac{\partial a}{\partial t}\right|_{\times}=-E_{\times}=\left(R_{\mu}^{-1} j\right)_{\times} .
$$

The reconnection rates have been normalized to the mean square fluctuation $\delta b_{r m s}^{2}(\sim 1$ for all the runs). Note that Eq. (3) gives exactly the reconnection rate for a fully 2D (MHD) case, while, in the HMHD case (2.5D), this expression gives the rate of component-reconnection. 

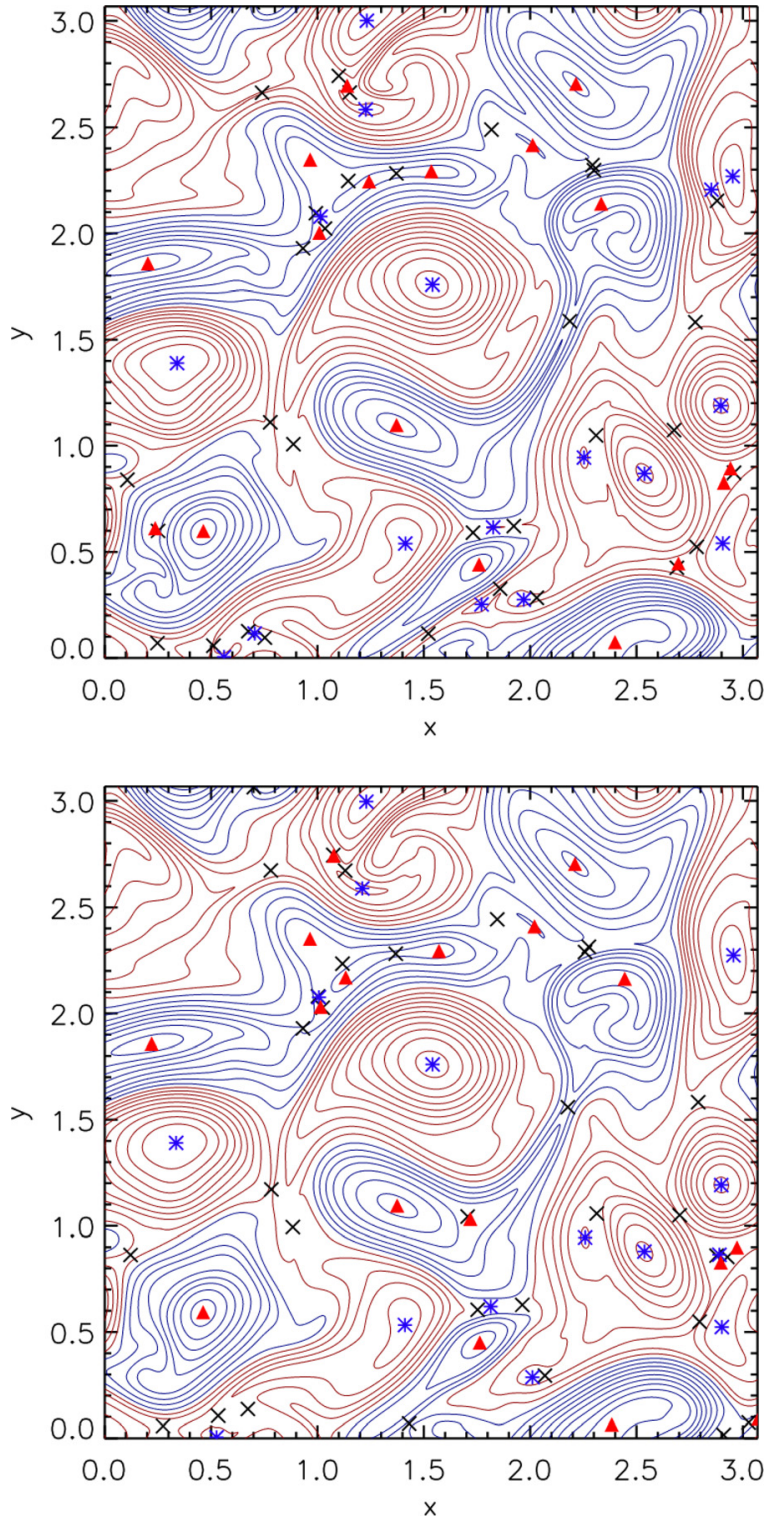

FIG. 4. Line-contours of the magnetic potential $a$ for the MHD case, run I (top), and for the HMHD case, run IV (bottom). In each, a sub-region of the simulation box is shown for clarity. The position of the critical points is reported as well: O-points (blue stars for the maxima and red diamonds for the minima) and X-points (black $\times$ ). Magnetic reconnection locally occurs at the $\mathrm{X}$-points.

The PDFs of $E_{\times}$, for the runs in Table I are reported in Fig. 6. All the distributions show a broad range with strong tails, the average lying near $E_{\times} \sim 0.05-0.06$ while the full range spans $\left|E_{\times}\right| \in\left[10^{-5}, 0.4\right]$. The PDFs have been constructed using constant weight $m$ per-bin (variable bin width), with $m=6$. The distribution of reconnection rates for the weak Hall case (run II, not shown) is very similar to the MHD results (run I), as expected from previous discussions. In the stronger Hall case (runs III and IV), instead, higher tails appear in the PDF. Apparently, for higher values of $\epsilon_{H}$ the frequency of occurrence of large reconnection rates is substantially increased. The increased frequency of large rates influences the means. As an example, for both runs I and II we obtained the mean value $\left\langle\left|E_{\times}\right|\right\rangle \simeq 0.05$, while for run IV $\left\langle\left|E_{\times}\right|\right\rangle \simeq 0.06$. This analysis confirms that the Hall term plays an important role in turbulence, where magnetic
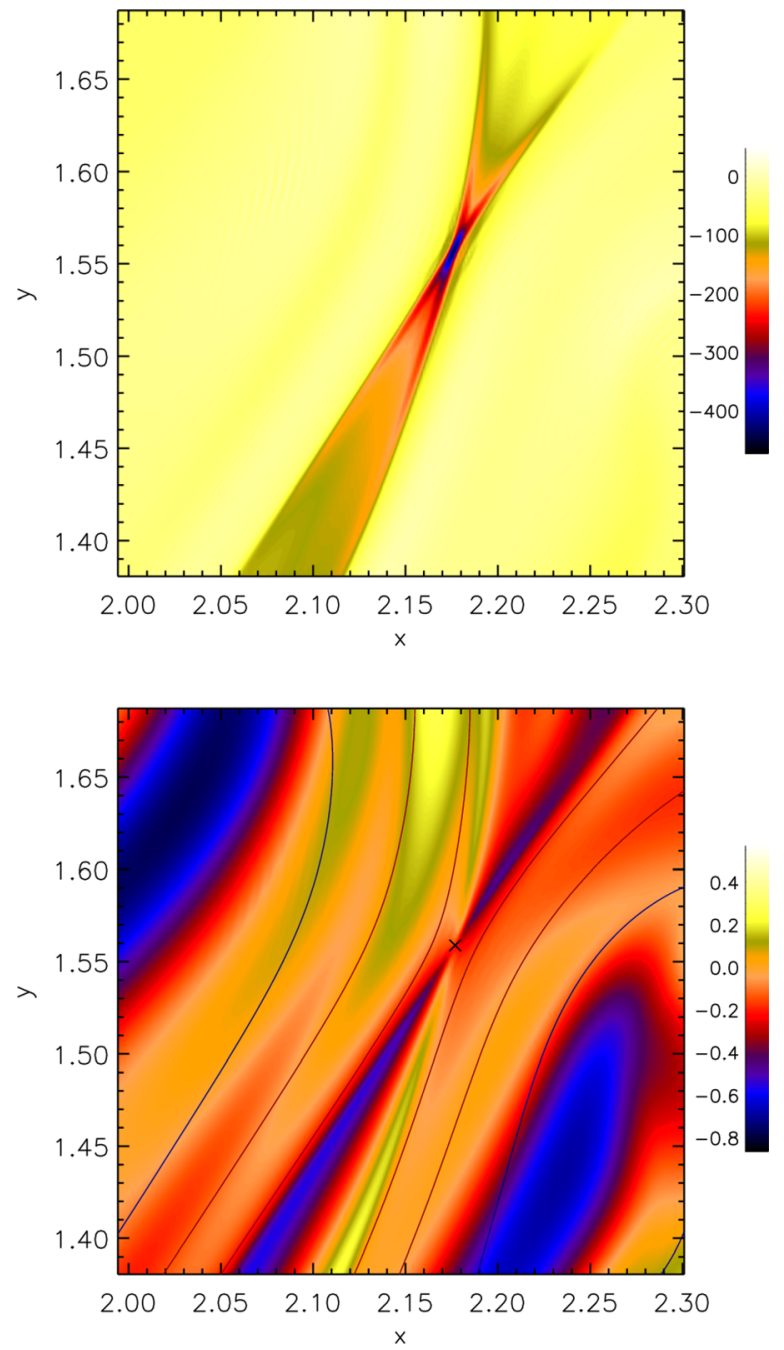

FIG. 5. (Top) A contour plot of the out-of-plane component of the current $j_{z}$ in a sub-region of the simulation box for run IV. The bifurcation of the sheet and the typical structure of a reconnection region are clearly visible. (Bottom) A contour plot of the out-of-plane component of the magnetic field $b_{z}$, in the same sub-region of the simulation box. The magnetic flux $a$ is also represented as a line contour. A quadrupole in the magnetic field can be identified, revealing the presence of Hall activity.

islands simultaneously reconnect in a complex way. In particular, when the Hall parameter is enhanced (increased ratio between the ion skin depth and the system size) distributions of reconnection rates have higher tails, revealing more frequently occurring explosive (very large) reconnection events than in the MHD case. As already pointed out in Ref. 5, there is a relation between the stronger reconnection rates and the geometry of the reconnection region, in fact these strong reconnecting electric fields satisfy the scaling relation,

$$
E_{\times} \sim \frac{l}{\delta} \sim \sqrt{\lambda_{R}}=\sqrt{\frac{\lambda_{\max }}{\lambda_{\min }}},
$$

where $l$ and $\delta$ are related, respectively, to the elongation and to the minimum thickness of the current sheet, i.e., to the geometry of the reconnecting region, while $\lambda_{\max }$ and $\lambda_{\min }$ are the Hessian eigenvalues evaluated at the X-point. In Fig. 7, we report the reconnection rates, associated with each X-point, as 


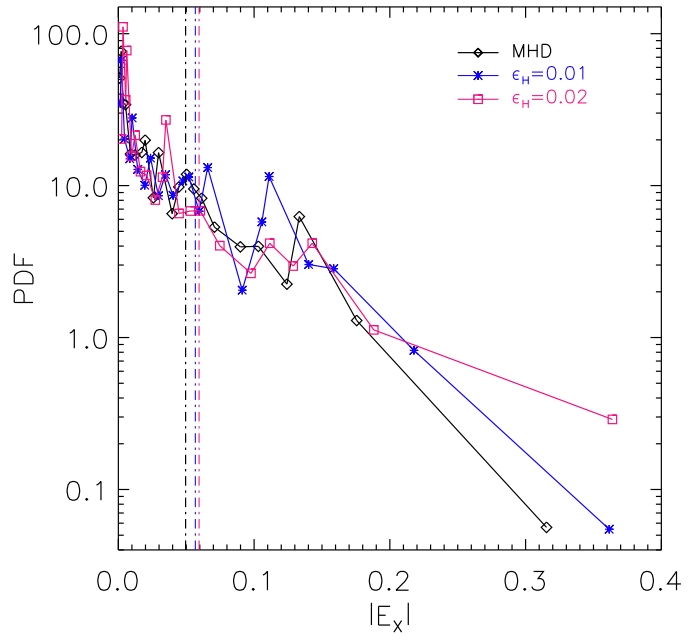

FIG. 6. PDF of the reconnection rates for $\epsilon_{H}=0$ (MHD, black rhombus), $\epsilon_{H}=1 / 100$ (blue stars), and $\epsilon_{H}=1 / 50$ (magenta squares). The vertical dashed-dotted lines represent the mean value of the distribution $\left\langle\left|E_{\times}\right|\right\rangle$for run I (black), run III (blue), and run IV (magenta).

a function of $\lambda_{R}$, for runs I, III, and IV. All distributions follow the proposed power-law (at least for stronger reconnection events), but in the HMHD case the values are more scattered and are bounded by lower $\lambda_{R}$. We evaluated, for each run, the distribution of $\left\langle\lambda_{R}\right\rangle$ as a function of $\epsilon_{H}$ (not shown here), and we observed that, for the strongest Hall effect case $\epsilon_{H}=1 / 50$, the computed value of $\left\langle\lambda_{R}\right\rangle$ is reduced to half the value obtained in the MHD case.

Following the methodology adopted in Ref. 5, to qualitatively characterize every reconnecting region, we extract information about currents, magnetic fields, and about the geometry of the diffusion regions. Since we know the ratio of the eigenvalues obtained from the Hessian matrix analysis, the problem reduces to find just one of these lengths, such as the current sheet thickness $\delta$. For this purpose, for each X-point, we build a system of reference that has the

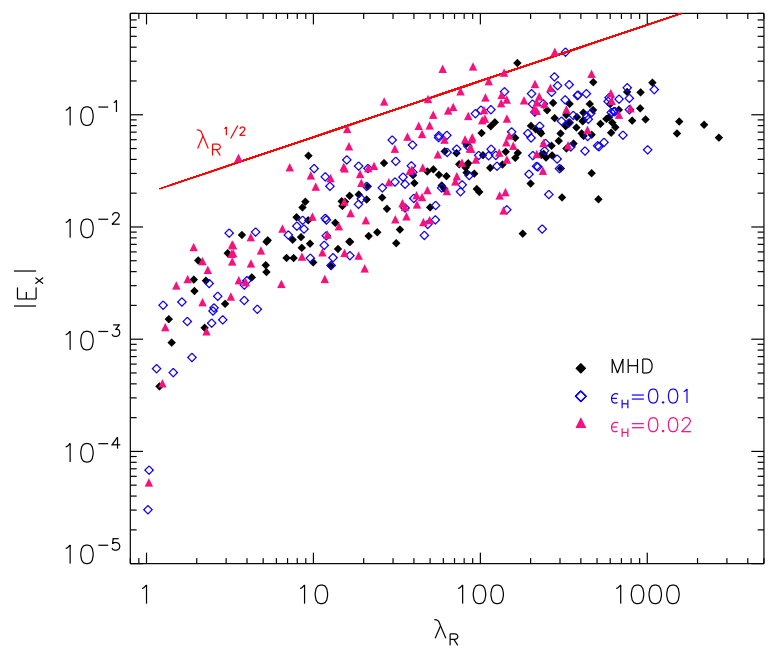

FIG. 7. The relation between the reconnection rate (the electric field at the $\mathrm{X}$-point) and the geometry of the reconnection region (the ratio of the eigenvalues) for both MHD (black rhombus) and HMHD (blue open rhombus and magenta triangles). The presence of a power-law fit (red solid line) demonstrates that there is a relation between the reconnection rate and the geometry of the diffusion region. origin at the X-point and, using the eigenvectors obtained in the Hessian analysis, we define a local coordinate system based on the unit vectors $\left\{\hat{e}_{s}, \hat{e}_{t}\right\}$, where the coordinates $s$ and $t$ are related to $\delta$ and $l$, respectively. With respect to this new reference system, the tangential and normal components of the magnetic field are evaluated as $b_{t}=\hat{e}_{t} \cdot \mathbf{b}$ and $b_{n}=\hat{e}_{s} \cdot \mathbf{b}$, while the current profile is obtained with an iterating fit procedure along the $s$ coordinate. Since the current profile is asymmetric (due to the asymmetric nature of turbulent reconnection), the thickness $\delta$ is determined as the sum of right-side and left-side thicknesses separately evaluated in the iterating procedure $\left(\delta=\delta_{1}+\delta_{2}\right)$. The above analysis has been performed only for stronger reconnection sites. For the present simulations, this means $\left|E_{\times}\right|>10^{-2}$ together with the restriction $\lambda_{\max } / \lambda_{\min }>100$.

In Fig. 8, we compare results from MHD (run I) and HMHD (run IV), showing the current profile and the local magnetic field near a particular X-point. The current density $j_{z}(s)$ and the projected tangential magnetic field $b_{t}(s)$ has been interpolated along the direction of $\hat{e}_{s}$. As already observed in Fig. 3, two main features are at work when the Hall effect is not negligible, namely, the thickness $\delta$ is reduced with respect to the MHD case, and $j_{z}$ reaches stronger values. This example serves to illustrate this effect, which we confirm statistically through an analysis of the values of $\delta$ and $l$ for all the stronger $\mathrm{X}$-point regions. The
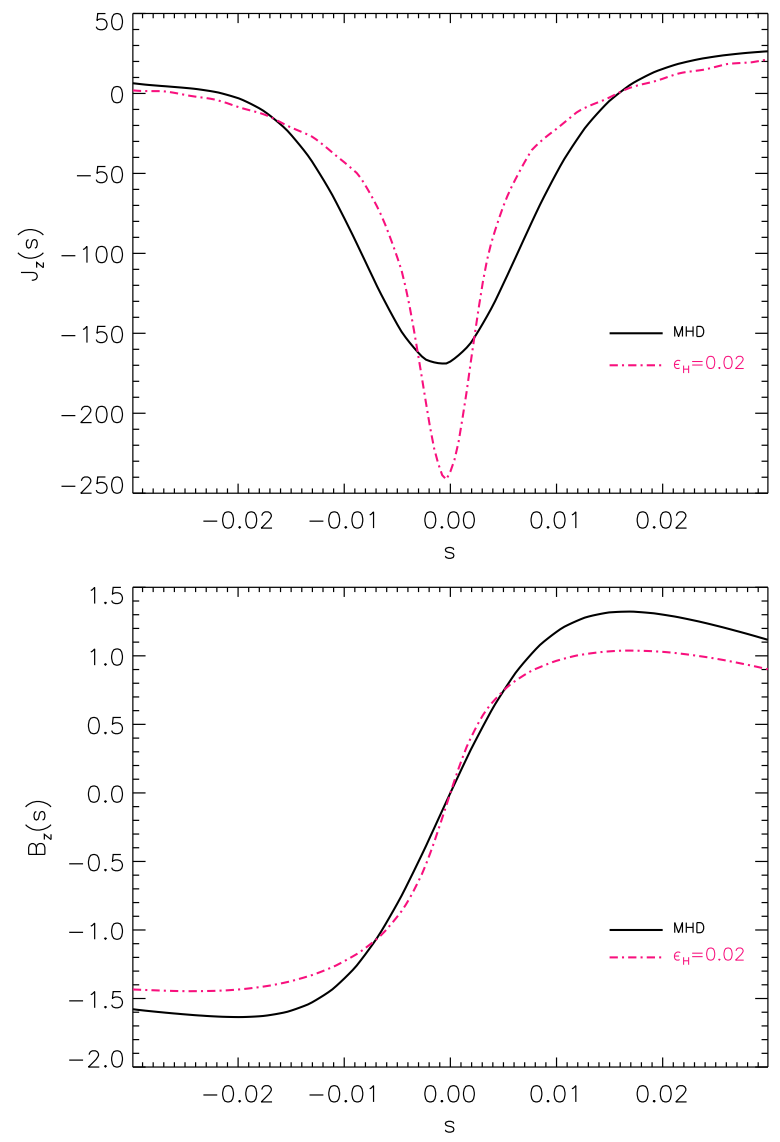

FIG. 8. Current density $j_{z}(s)$ (top) and tangential component of the magnetic field $b_{t}$ (bottom), in the vicinity of the same X-point, for both MHD (black line) and HMHD with $\epsilon_{H}=1 / 50$ (magenta dashed-dotted line). $s$ is the direction along $\hat{e}_{s}$-the steepest gradient of the Hessian of $a$. In HMHD, current sheets are narrower and more intense. 

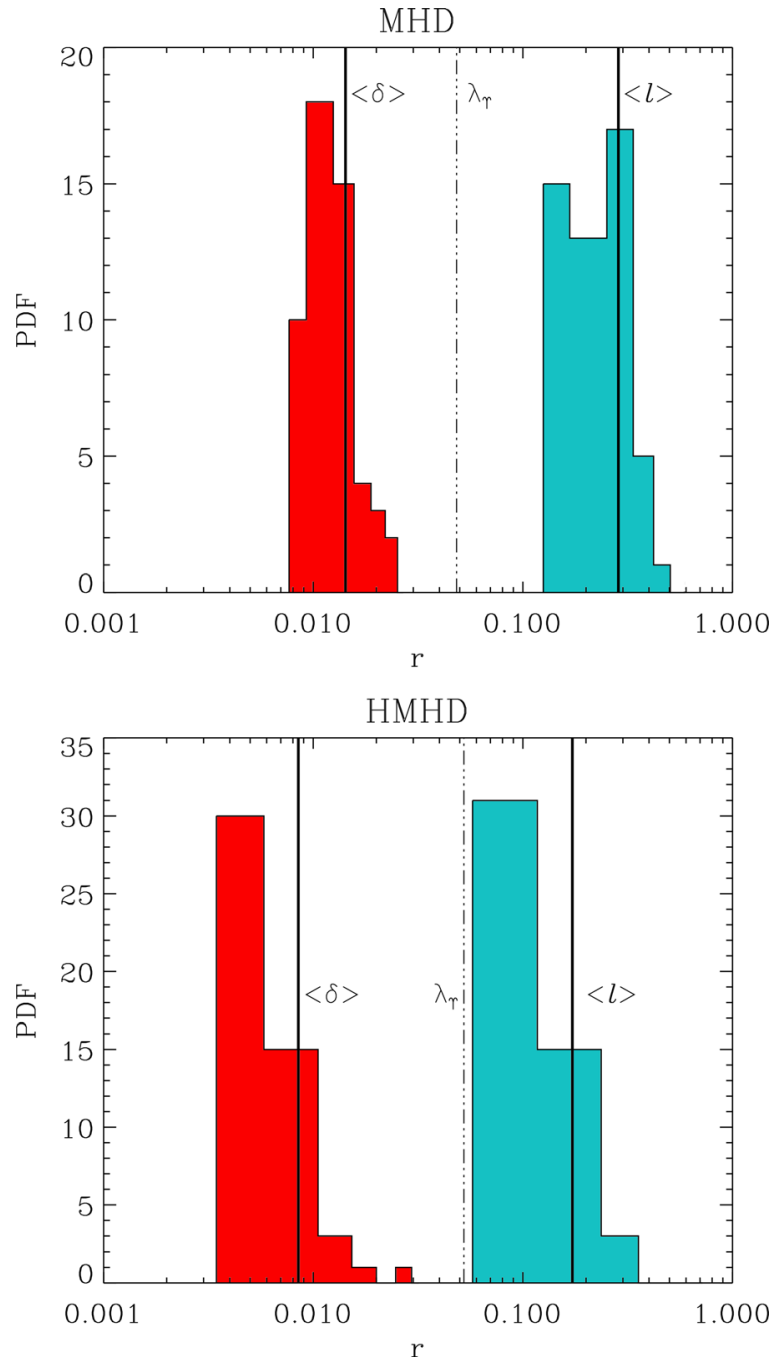

FIG. 9. Histograms of thicknesses $\delta$ (red bars) and elongations $l$ (azure bars) for MHD (top) and HMHD with $\epsilon_{H}=1 / 100$ (bottom). Vertical lines are average values $\langle\delta\rangle$ (red) and $\langle l\rangle$ (azure), the vertical dashed-dotted line represents the Taylor microscale $\lambda_{T}$. The distributions are shifted towards smaller values for the HMHD case.

associated PDFs of $\delta$ and $l$ have been computed, for both MHD and HMHD, and the comparison is reported in Fig. 9. In the Hall case, the distributions are shifted towards smaller values and, accordingly, on average the current sheets are both thinner and shorter than in the MHD counterpart. These characteristic average lengths are reported in Table II.

Recently, ${ }^{4,5}$ it was demonstrated that the fastest reconnection events in resistive MHD turbulence can be described by a modified Sweet-Parker theory which takes into account asymmetries in the reconnecting magnetic field. ${ }^{27}$ This model predicts that the reconnection rate for incompressible resistive plasmas scale as

$$
E_{\times}^{t h}=\sqrt{\frac{b_{1}^{3 / 2} b_{2}^{3 / 2}}{R_{\mu} l}},
$$

where $b_{1}$ and $b_{2}$ are the upstream magnetic fields on each side of the X-point. For this purpose the magnetic fields are evaluated at $2 \delta_{1,2}$, namely $b_{i}=b_{t}\left(2 \delta_{i}\right)$. The comparison between run I (MHD), run III $\left(\epsilon_{H}=1 / 100\right)$ and run IV
TABLE II. Characteristic lengths and reconnection rates for each run. The first column is the Hall parameter, second column the average thickness of reconnection regions, the average length of reconnection sites is reported in column 3, while the average and the maximum reconnection rates are reported in columns 4 and 5 , respectively.

\begin{tabular}{lccccc}
\hline \hline Run & $\epsilon_{H}$ & $\langle\delta\rangle$ & $\langle l\rangle$ & $\left\langle E_{\times}\right\rangle$ & $\operatorname{Max}\left\{E_{\times}\right\}$ \\
\hline I & 0 & 0.014 & 0.286 & 0.049 & 0.315 \\
II & $1 / 400$ & 0.013 & 0.272 & 0.050 & 0.326 \\
III & $1 / 100$ & 0.008 & 0.172 & 0.057 & 0.362 \\
IV & $1 / 50$ & 0.005 & 0.077 & 0.059 & 0.364 \\
\hline \hline
\end{tabular}

$\left(\epsilon_{H}=1 / 50\right)$ is reported in Fig. 10. It is apparent that in Hall cases the reconnection rates are faster and more broadly distributed than the MHD prediction. In particular, note the constant fractional increase in reconnection rate $\left|E_{\times}\right|$with increasing Hall parameter $\epsilon_{H}$. To understand why the HMHD reconnection data does not follow the prediction of resistive MHD, note that a number of authors have recently shown that resistive HMHD reconnection does not follow the standard Sweet-Parker scaling. ${ }^{28,29}$ However, it was argued that reconnection in resistive HMHD is not a stable reconnection configuration. ${ }^{30}$ When the current sheet is thicker than $d_{i}$, reconnection rates follow the Sweet-Parker prediction and scale as $\eta^{1 / 2}$. When the current sheet is thinner than $d_{i}$, the process is faster and reconnection rates scale linearly in $\eta\left(E_{\times} \propto \eta\right)$. Thus, to see if the reconnection in resistive HMHD turbulence follows the predictions of a steady-state asymmetric reconnection model (as it does in resistive $\mathrm{MHD}^{4,5}$ ) we have to apply the more general form of the prediction of asymmetric reconnection which should apply independent of dissipation mechanism. ${ }^{27}$ The prediction is that the reconnection electric field at the X-point should scale as

$$
E_{\times}^{t h} \sim \frac{\left(b_{1} b_{2}\right)^{3 / 2}}{b_{1}+b_{2}} \frac{2 \delta}{l}
$$

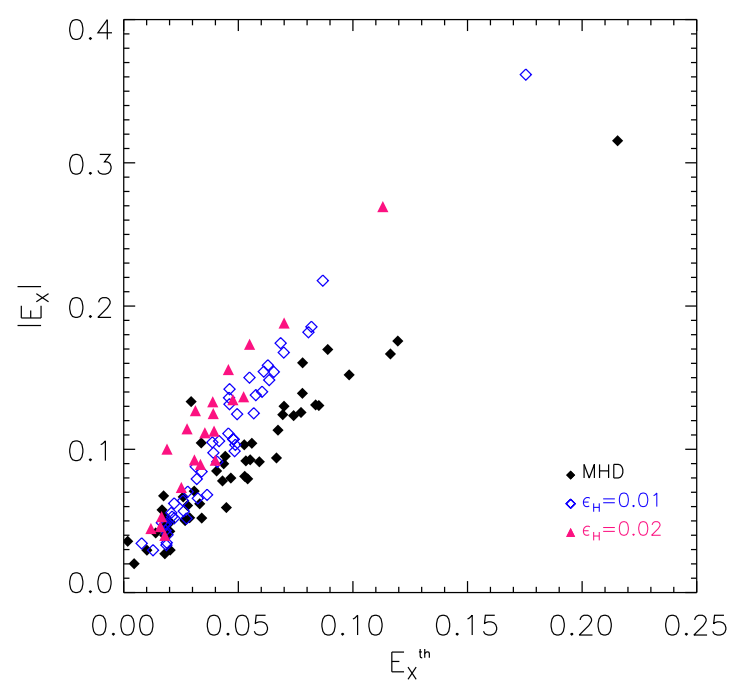

FIG. 10. Computed reconnection rates vs expectation from Eq. (5), for runs I, III, and IV. The Hall cases seem to slightly depart from the Sweet-Parker asymmetric expectation. 


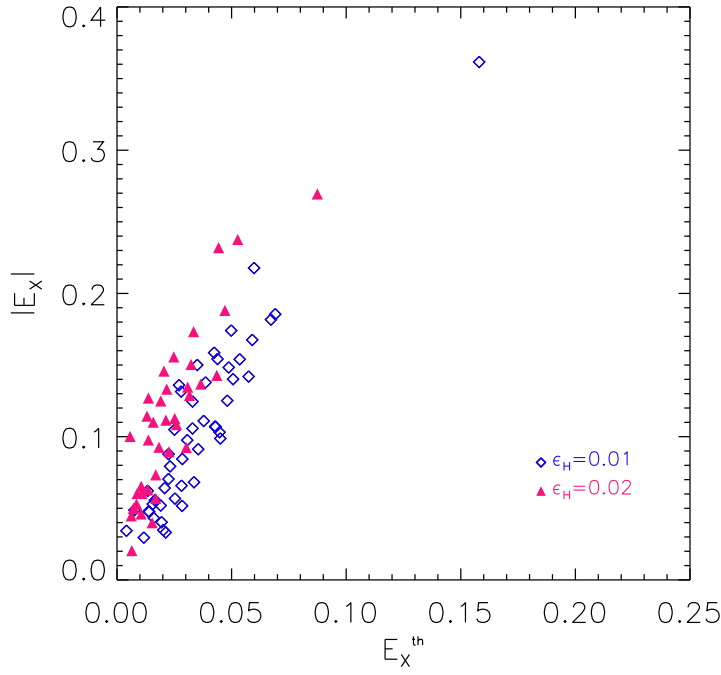

FIG. 11. Computed reconnection rates vs expectation from Eq. (6), for runs III and IV. Strongest reconnection rates in the Hall MHD cases scale linearly with expected values.

which replaces Eq. (5). A comparison of the measured and predicted reconnection rates for the strongest HMHD reconnection events in run III $\left(\epsilon_{H}=1 / 100\right)$ and run IV $\left(\epsilon_{H}=1 / 50\right)$ is plotted in Fig. 11.

The prediction is seen to collapse the data towards a line to a certain degree, which is suggestive that the reconnection agrees with the models. However, we note that the agreement is not as strong as for the resistive MHD case. ${ }^{4,5}$ One possible reason that the agreement is not as good is if the resistive HMHD reconnection is an unstable branch, it will not stay in a steady state for any extended time. A full test of whether Hall reconnection models describe reconnection in a turbulent system should employ hyper-resistive and/or electron inertia terms so that reconnection is in a stable mode. ${ }^{30}$

\section{CONCLUSIONS}

We have provided a direct comparison of the statistics of reconnection rates obtained from simulations of MHD turbulence and Hall MHD turbulence for cases with increasing Hall parameter $\epsilon_{H}=d_{i} / L_{0}$. For small values of Hall parameter there is very little difference in distributions of electric current density or reconnection rates. However for stronger Hall parameter $\epsilon_{H}>0.01$ one begins to see enhancements of reconnection. In particular while there is a modest increase in average reconnection rate, there is a more dramatic increase in the frequency of occurrence of large reconnection rates. Associated with this is the shortening and thinning of current sheets, and the appearance of bifurcated current sheets, all previously reported as properties of isolated laminar reconnection sites with Hall effect. Evidently the impact of Hall effect depends crucially on whether this term in Ohm's Law become significant at wavenumbers $k_{H}$ lower than the reciprocal dissipation scale $k_{\text {diss }}$, so that it influences the upper inertial range, or if it becomes significant only at scales smaller than where dissipation becomes strong. Therefore in HMHD simulations with scalar resistivity and viscosity such as the ones we carried out, the simulator has complete control over the relationship of the relevant wavenumbers $k_{H}$ and $k_{\text {diss }}$. Using this flexibility in simulation to independently vary strength of the Hall effect, the dissipation scale, and the large scale energy budget, prior studies have provided some insight into the relationship of these effects. For example in Ref. 33, Matthaeus et al. examined the influence of varying $\epsilon_{H}$ $\left(\epsilon_{H}=0,1 / 32,1 / 16, \ldots 1\right)$ on energy decay rates while Reynolds numbers were fixed at values of 400 or 1000 . Little effect on decay rate was seen until $\epsilon_{H} \sim 1$.

On the other hand, in the related problem of the effects of small scale MHD turbulence on a large scale reconnection simulation, in Ref. 15 it was found that the influence of Hall effect becomes comparable to that of turbulence when $\epsilon_{H} \approx 1 / 9$. Here we have examined the distribution of reconnection rates, which is related to the physics of the cascade in a complex and incompletely understood way, and find that the Hall effect begins to produce measurable changes in the distribution of rates at values of $\epsilon_{H} \sim[0.01-0.02]$. Evidently reconnection rates are more sensitive to Hall effect than is the overall cascade rate. Simulation has provided some insights into these relationships, but it is clear that more complete understanding will require further study. What is less clear is how to estimate this relationship of dissipation and Hall effect in a low collisionality plasma. Typically, ${ }^{31,32}$ kinetic theory suggests that $d_{i}$ is near the scale at which dissipative effects become significant, but it is not clear to us whether one can make general statements concerning the precise value of the ratio $k_{H} / k_{\text {diss }}$. If dissipation sets in at scales much smaller than $d_{i}$, e.g., through dominance of electron dissipation effects, the present work suggests that the Hall effect can be important in establishing the most robust reconnection rates that will be observed in turbulence. We have not however examined cases with very large Hall parameters $\epsilon_{H} \sim 1$, which become computationally prohibitive.

This research was supported in part by Marie Curie Project FP7 PIRSES-2010-269297_-“Turboplasmas,” POR Calabria FSE 2007/2013, NASA Heliophysics Theory Program NNX11AJ44G, NSF Solar terrestrial Program AGS-1063439, UBACYT 20020090200602, PICT/ANPCyT 2007-00856, PIP/CONICET 11220090100825, the NASA MMS mission NNX08AT76G, and the NSF Grant PHY-0902479.

\section{APPENDIX: ABOUT THE ACCURACY IN HALL MHD TURBULENCE}

In Ref. 34, it was suggested that oversampling the Kolmogorov dissipation scale by a factor of 3 allows accurate computation of the kurtosis, the scale-dependent kurtosis, and the reconnection rates, in the case of MHD 2D simulations. In particular, the proposed tests stated the conditions on spatial resolution that must be attained to accurately compute the tail of the distribution of reconnection rates, because this tail measures the likelihood of the highest rates of reconnection. The assumption is that accurate computations of fourth order moments gives rise to accurate computation of reconnection rates. Here we want to examine the validity of the test in the Hall MHD case. We report a numerical example, where we compare run IV with another run, with 
TABLE III. Parameters for the simulations. The initially excited Fourier modes have $4<|k|<10$, and $k_{\text {diss }}$ means the maximum value of $k_{\text {diss }}(t)$.

\begin{tabular}{lccccc}
\hline \hline Run & Grid & $R_{\mu}\left(R_{\nu}\right)$ & $k_{\max }$ & $k_{\text {diss }}$ & $\frac{k_{\text {max }}}{k_{\text {diss }}}$ \\
\hline I & $2048^{2}$ & 1700 & 684 & 179 & 3.8 \\
II & $4096^{2}$ & 1700 & 1365 & 179 & 7.6 \\
\hline \hline
\end{tabular}

identical initial data and dissipation coefficients, but half spatial resolution (the list of parameters for both simulations is reported in Table III).

As already observed in Ref. 35 but not shown here, if simulations differ only in resolutions, the spectra agree well, the curves nearly overlay each other over the full range of overlapping $k$, with only small discrepancies in the lower resolution run near its maximum retained wavenumber. In Fig. 12, the PDFs of $\left|E_{\times}\right|$together with their respective error bars are shown. The curves agree very well out to 0.1 , but then, near the upper end, the PDFs differ by a factor of $\sim 2$. In addition, we report in Fig. 13 the contour lines of the magnetic potential $a$ together with the positions of reconnection sites, for both runs ( $2048^{2}$ top, $4096^{2}$ bottom) in a sub-region of the simulation box. In the lower-resolved case, a higher number of X-points is present. Different than the MHD case, where for $k_{\max } / k_{\text {diss }} \sim 3$ a clear saturation in $C_{\times}$occurs, in the HMHD case this condition was not achieved $\left(C_{\times}=168\right.$ for run I and $C_{\times}=126$ for run II). Following Ref. 34, such a factor discrepancy places the lower resolved simulation down at around an effective resolution of $750^{2}$, and the higher resolved one down to $1536^{2}$, but still at good level of accuracy. We believe that the spatial resolutions of the simulations in the main text are adequate to support the physical conclusions that we report. It is clear that the issue of truly convergent results for turbulent reconnection in Hall MHD is an even more difficult problem than it is for resistive

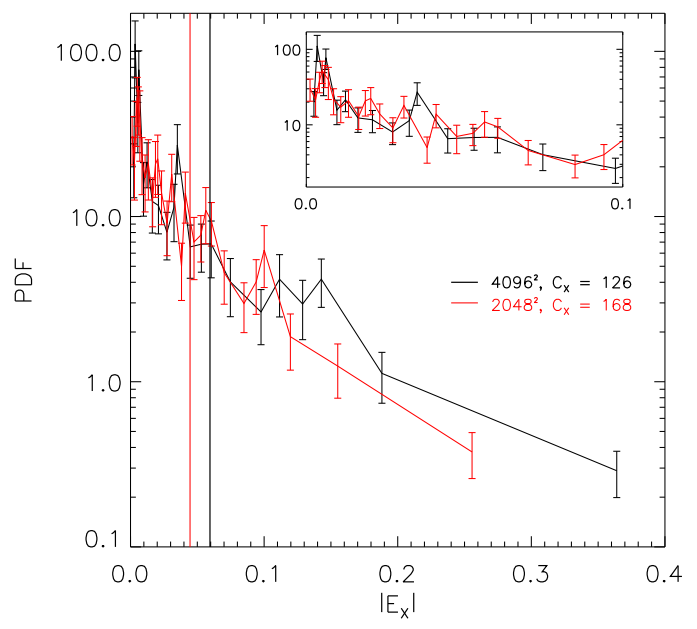

FIG. 12. PDFs of the reconnection rate $\left|E_{\times}\right|$for run 1 (red line) and run 2 (black line) (Table III) with their respective error bars. The curves agree very well up to 0.1 (as better shown in the insert), but then they are separated. In the label are also reported the number of X-points for both runs. The vertical dashed-dotted lines represent the mean value of the distribution $\left\langle\left|E_{\times}\right|\right\rangle$for run 1 (red) and run 2 (black).
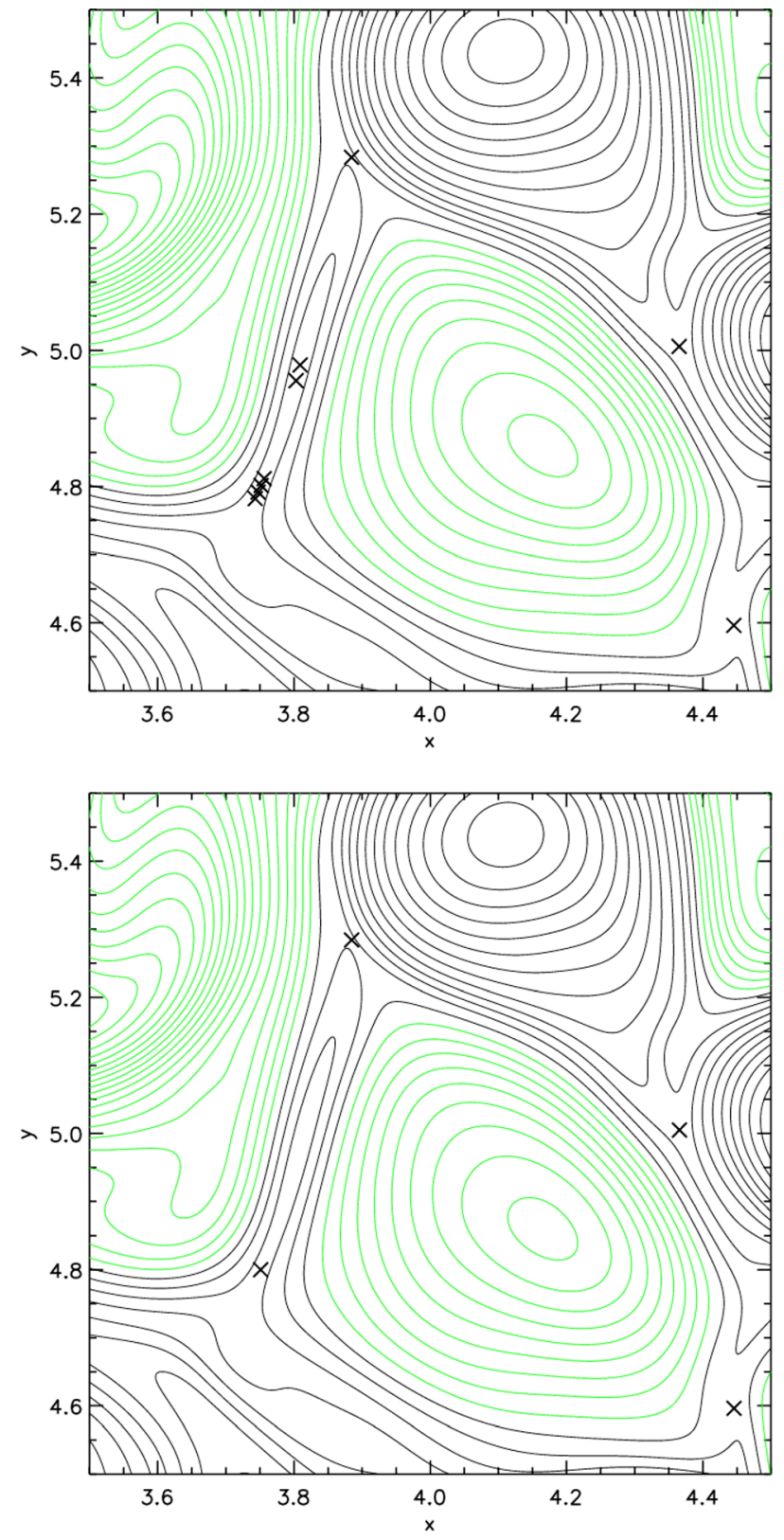

FIG. 13. Contour lines of the magnetic potential $a$ with the positions of reconnection sites (X-points) in black $\times$ from run 1 (top) and run 2 (bottom).

MHD. ${ }^{34}$ At present, we call attention to this problem, which has largely been undocumented in the reconnection literature even for laminar cases.

${ }^{1}$ B. U. O. Sonnerup, J. Plasma Phys. 4, 161 (1970).

${ }^{2}$ V. M. Vasyliunas, Rev. Geophys. Space Phys. 13, 303, doi:10.1029/ RG013i001p00303 (1975).

${ }^{3}$ E. N. Parker, Astrophys. J. 264, 642 (1983).

${ }^{4}$ S. Servidio, W. H. Matthaeus, M. A. Shay, P. A. Cassak, and P. Dmitruk, Phys. Rev. Lett. 102, 115003 (2009).

${ }^{5}$ S. Servidio, W. H. Matthaeus, M. A. Shay, P. Dmitruk, P. A. Cassak, and M. Wan, Phys. Plasmas 17, 032315 (2010).

${ }^{6}$ D. Biskamp, Nonlinear Magnetohydrodynamics (Cambridge University Press, Cambridge, England, 1993).

${ }^{7}$ W. H. Matthaeus and S. L. Lamkin, Phys. Fluids 29, 2513 (1986).

${ }^{8}$ B. U. O. Sonnerup, in Solar System Plasma Physics, edited by E. N. Parker, C. F. Kennel, and L. J. Lanzerotti (North-Holland, 1979), pp. $47-102$.

${ }^{9}$ K. V. Roberts and J. B. Taylor, Phys. Rev. Lett. 8, 197 (1962). 
${ }^{10}$ S. Servidio, V. Carbone, L. Primavera, P. Veltri, and K. Stasiewicz, Planet. Space Sci. 55, 2239 (2007).

${ }^{11}$ S. Galtier and E. Buchlin, Astrophys. J. 656, 560 (2007).

${ }^{12}$ P. D. Mininni, A. Alexakis, and A. Pouquet, J. Plasma Phys. 73, 377 (2007).

${ }^{13}$ W. H. Matthaeus, S. Servidio, and P. Dmitruk, Phys. Rev. Lett. 101, 149501 (2008).

${ }^{14}$ P. Dmitruk and W. H. Matthaeus, Phys. Plasmas 13, 042307 (2006).

${ }^{15}$ Z. W. Ma and A. Bhattacharjee, J. Geophys. Res. 106, 3773, doi:10.1029/ 1999JA001004 (2001).

${ }^{16}$ J. Birn, J. F. Drake, M. A. Shay, B. N. Rogers, R. E. Denton, M. Hesse, M. Kuznetsova, Z. W. Ma, A. Bhattacharjee, A. Otto, and P. L. Prichett, J. Geophys. Res. 106, 3715, doi:10.1029/1999JA900449 (2001).

${ }^{17}$ D. Smith, S. Ghosh, P. Dmitruk, and W. H. Matthaeus, Geophys. Res. Lett. 31, L02805, doi:10.1029/2003GL018689 (2004).

${ }^{18}$ Q. Lu, C. Huang, J. Xie, R. Wang, M. Wu, A. Vaivads, and S. Wang, J. Geophys. Res. 115, A11208, doi:10.1029/2010JA015713 (2010).

${ }^{19}$ P. A. Cassak, M. A. Shay, and J. F. Drake, Phys. Rev. Lett. 95, 235002 (2005).

${ }^{20}$ P. A. Cassak, J. F. Drake, M. A. Shay, and B. Eckhardt, Phys. Rev. Lett. 98, 215001 (2007).

${ }^{21}$ S. Servidio, W. H. Matthaeus, and V. Carbone, Phys. Plasmas 15, 042314 (2008).

${ }^{22}$ M. Wan, S. Oughton, S. Servidio, and W. H. Matthaeus, Phys. Plasmas 16, 080703 (2009)
${ }^{23}$ M. A. Shay, J. F. Drake, R. E. Denton, and D. Biskamp, J. Geophys. Res. 103, 9165, doi:10.1029/97JA03528 (1998).

${ }^{24}$ A. Retinò, D. Sundkvist, A. Vaivads, F. Mozer, M. André, and C. J. Owen, Nat. Phys. 3, 236 (2007).

${ }^{25}$ Y. Ren, M. Yamada, S. Gerhardt, H. Ji, R. Kulsrud, and A. Kuritsyn, Phys. Rev. Lett. 95, 055003 (2005).

${ }^{26}$ W. H. Matthaeus, C. D. Cothran, M. Landreman, and M. R. Brown, Geophys. Res. Lett. 32, L23104, doi:10.1029/2005GL023973 (2005).

${ }^{27}$ P. A. Cassak and M. A. Shay, Phys. Plasmas 14, 102114 (2007).

${ }^{28}$ L. Chacón, A. Simakov, and A. Zocco, Phys Rev. Lett. 99, 235001 (2007).

${ }^{29}$ L. M. Malyshkin, Phys. Rev. Lett. 101, 225001 (2008).

${ }^{30}$ P. A. Cassak, M. A. Shay, and J. F. Drake, Phys. Plasmas 17, 062105 (2010).

${ }^{31}$ S. P. Gary and J. E. Borovsky, J. Geophys. Res. 109, A06105, doi:10.1029/2004JA010399 (2004).

${ }^{32}$ T. N. Parashar, S. Servidio, B. Breech, M. A. Shay, and W. H. Matthaeus, Phys. Plasmas 17, 102304 (2010).

${ }^{33}$ W. H. Matthaeus, P. Dmitruk, D. Smith, S. Ghosh, and S. Oughton, Geophys. Res. Lett. 30, 2104, doi:10.1029/2003GL017949 (2003).

${ }^{34}$ M. Wan, S. Oughton, S. Servidio, and W. H. Matthaeus, Phys. Plasmas 17, 082308 (2010).

${ }^{35}$ S. Servidio, P. Dmitruk, A. Greco, M. Wan, S. Donato, P. A. Cassak, M. A. Shay, V. Carbone, and W. H. Matthaeus, Nonlinear Processes Geophys. 18, 675 (2011). 